\title{
Comparación de los niveles de procalcitonina por microorganismo en niños con sepsis
}

\section{Comparison of procalcitonin levels by microorganism in children with sepsis}

\author{
José Iván Castillo-Bejarano,* Agustín De Colsa-Ranero, ${ }^{\ddagger}$ Oscar Tamez-Rivera, ${ }^{\S}$ \\ Andrés Guillén-Lozoya,* Napoleón González-Saldaña, ${ }^{\S}$ Alfonso Huante-Anaya," \\ Daniel Siller-Rodríguez," Abiel Homero Mascareñas-de los Santos* \\ * Departamento de Pediatría, División de Infectología, Hospital Universitario «Dr. José Eleuterio González». Monterrey, México. \\ ₹ Laboratorio de Biología Molecular, Instituto Nacional de Pediatría (INP). Ciudad de México, México. \\ § Departamento de Infectología, Instituto Nacional de Pediatría (INP). Ciudad de México, México. \\ " Departamento de Nefrología, Instituto Nacional de Pediatría (INP). Ciudad de México, México \\ " Unidad de Vigilancia Epidemiológica, Christus Muguerza Hospital Alta Especialidad. Monterrey, México.
}

\section{RESUMEN}

Introducción: El diagnóstico oportuno en sepsis es crucial para incrementar la tasa de supervivencia. Estudios previos han identificado en la procalcitonina una herramienta con el potencial de predecir la positividad de hemocultivos en pacientes con bacteriemia; sin embargo, los estudios se han realizado en adultos. El objetivo de este estudio fue analizar los niveles de procalcitonina (PCT) por especie en infecciones del torrente sanguíneo (ITS) en niños y evaluar su utilidad en pacientes inmunocomprometidos. Material y métodos: Se revisaron expedientes clínicos de menores de 18 años ingresados de 2011 a 2018. Se incluyeron pacientes con diagnóstico de sepsis y con PCT en las 72 horas previas a la toma de un hemocultivo. Se usó la prueba de KruskalWallis para comparar las diferencias entre grupos y curvas ROC para evaluar los niveles de corte de PCT. Para el análisis estadístico se utilizó SPSS v. 21. Resultados: 120 pacientes fueron incluidos. La edad media fue de 55 meses. Los niveles medios de PCT en inmunocomprometidos fue $26.68 \mu \mathrm{g} / \mathrm{L}$, en comparación con $8.78 \mu \mathrm{g} / \mathrm{L}$ en el grupo de inmunocompetentes. Los pacientes con bacteriemia por bacilos Gram negativos (BGN) tuvieron la media más alta de PCT $(18.2 \pm 34.2, p<0.001)$. La sensibilidad y especificidad fue de 78 y $53 \%$ para cocos Gram positivos (CGP), 60.9 y $33.3 \%$ para BGN y 75 y $25 \%$ para levaduras, respectivamente. El análisis de subgrupos demostró que la PCT tenía una

\section{ABSTRACT}

Introduction: Timely diagnosis and accurate identification of the causative microorganism in sepsis is crucial to offer targeted treatment and increase survival rates. Previous studies have aimed to identify biomarkers that could potentially predict blood culture positivity in patients with bacteremia; however, most of the research has been performed in adult populations. This study aimed to analyze procalcitonin (PCT) levels in confirmed bloodstream infections by species in children and assess their utility in immunocompromised patients. Material and methods: Medical records of children younger than 18 years admitted from 2011 to 2018 were reviewed. Subjects who met the diagnostic criteria for sepsis with PCT levels collected within 72 hours before obtaining a blood culture were included. Kruskal-Wallis test was used to compare differences among groups. Receiver-operating characteristic (ROC) curves were used to evaluate PCT cut-offs. SPSS v. 21 was used for statistical analysis. Results: A total of 120 patients were included. Mean age was 55 months. Mean PCT levels in immunosuppressed patients was $26.68 \mu \mathrm{g} / \mathrm{L}$, compared to $8.78 \mu \mathrm{g} / \mathrm{L}$ in the immunocompetent group. Subjects with bacteremia by Gram negative bacilli (GNB) had the highest mean PCT levels (18.2 \pm 34.2$)(p<0.001)$. Sensitivity and specificity were $78 \%$ and $53 \%$ for Gram positive cocci (GPC), $60.9 \%$ and $33.3 \%$ for GNB, and $75 \%$ and $25 \%$ for yeasts, respectively.

Abreviaturas:

$\mathrm{PCT}=$ Procalcitonina .

$\mathrm{BGN}=$ Bacilos Gram negativo.

CGP $=$ Cocos Gram positivos.

ITS = Infección del torrente sanguíneo.

$\mathrm{CVC}=$ Catéter venoso central.

SRIS = Síndrome de respuesta inflamatoria sistémica.

ROC = Característica operativa del receptor (ROC, por sus siglas en inglés: receiver-operating characteristic).

$\mathrm{AUC}=$ Área bajo la curva.

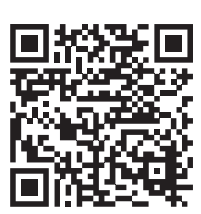

Citar como: Castillo-Bejarano Jl, De Colsa-Ranero A, Tamez-Rivera O, Guillén-Lozoya A, González-Saldaña N, Huante-Anaya A et al. Comparación de los niveles de procalcitonina por microorganismo en niños con sepsis. Rev Latin Infect Pediatr. 2021; 34 (1): 27-33. https://dx.doi.org/10.35366/99825 Financiamiento: Ninguno.

Conflicto de intereses: Ninguno. 
sensibilidad de $87.5 \%$ y especificidad de $16.7 \%$ para predecir bacteriemia por BGN en niños inmunodeficientes. Conclusiones: La PCT puede ser considerada como un biomarcador sustituto en niños inmunocomprometidos y como una herramienta útil para diferenciar la etiología por especies.

Palabras clave: Procalcitonina, sepsis, sepsis bacteriana.

\section{INTRODUCCIÓN}

La sepsis es una de las principales causas de mortalidad en el mundo. La identificación oportuna y precisa del agente etiológico es crucial para lograr incrementar la tasa de supervivencia mediante un tratamiento específico. Estudios previos han explorado el uso de biomarcadores que podrían predecir la positividad de los hemocultivos en pacientes con bacteriemia; sin embargo, la mayor parte de la evidencia disponible se ha enfocado en población adulta. ${ }^{1,2}$

La procalcitonina (PCT) es una prohormona de 116 aminoácidos sintetizada y secretada principalmente por las células $\mathrm{C}$ tiroideas; sin embargo, cualquier parénquima es capaz de secretarla durante una infección. ${ }^{3-5}$ Se pueden observar niveles elevados de PCT en pacientes críticos. ${ }^{6-10}$ Se ha reportado que los niveles elevados de PCT predicen la positividad de hemocultivos en adultos con bacteriemia confirmada por hemocultivo, con una sensibilidad de $75 \%$ y especificidad de $72 \% .{ }^{9}$ Asimismo, se ha descrito un valor predictivo negativo elevado (95.4\%) para predecir bacteriemia con niveles normales de PCT. ${ }^{10}$

La PCT puede ser utilizada como una herramienta que diferencia entre posibles etiologías bacterianas, particularmente bacilos Gram negativos (BGN) y cocos Gram positivos (CGP). ${ }^{11}$ Existe una brecha de conocimiento en la utilidad diagnóstica de los niveles de PCT en niños con infecciones del torrente sanguíneo, especialmente en inmunocomprometidos. El objetivo de este estudio fue analizar los niveles de PCT en niños con sepsis y evaluar su utilidad para diferenciar entre especies bacterianas.

\section{MATERIAL Y MÉTODOS}

Este estudio se llevó a cabo en el Instituto Nacional de Pediatría (INP) en la Ciudad de México durante el periodo de 2011 al 2018. Se incluyeron sujetos menores de 18 años que cumplieran con criterios diagnósticos de sepsis y que contaran con niveles de PCT tomados dentro de las 72 horas previas a la realización de un hemocultivo.
Subgroup analysis showed $87.5 \%$ sensitivity and $16.7 \%$ specificity of PCT for predicting documented GNB bacteremia in immunodeficient children. Conclusions: PCT may be considered as a surrogate biomarker in immunocompromised children and a viable tool to differentiate etiology by species.

Keywords: Procalcitonin, sepsis, bacterial sepsis.

Los sujetos con hemocultivos polimicrobianos (20 más microorganismos) y con aislamiento de bacterias comensales (estafilococos coagulasa-negativos, bacilos Gram positivos y Micrococcus spp.) en un hemocultivo periférico fueron excluidos. Se incluyeron hemocultivos con crecimiento de bacterias comensales de pacientes con una infección de torrente sanguíneo asociada con catéter venoso central y en casos de aislamientos del mismo microorganismo en dos o más hemocultivos periféricos.

Las muestras fueron procesadas mediante el sistema automatizado de hemocultivos BD BACTEC ${ }^{\mathrm{TM}}$. La identificación bacteriana y las pruebas de susceptibilidad se realizaron mediante BD Phoenix ${ }^{\mathrm{TM}} 100$. Los niveles de PCT se obtuvieron con el sistema Thermo Fisher Scientific ${ }^{\mathrm{TM}}$, con un valor de corte de $0.5 \mu \mathrm{g} / \mathrm{L}$.

La sepsis se definió como la presencia de síndrome de respuesta inflamatoria sistémica (SRIS) con infección documentada mediante hemocultivo según la Society of Critical Care Medicine. ${ }^{4}$ Los niños que cumplieron con dos o más de los siguientes criterios se diagnosticaron con SRIS: fiebre $\geq 38^{\circ} \mathrm{C}$, hipotermia $<36{ }^{\circ} \mathrm{C}$, taquicardia $>90$ latidos por minuto, taquipnea $>20$ respiraciones por minuto, hipocapnia $\mathrm{PaCO}_{2}<32 \mathrm{mmHg}$ y leucocitosis/leucopenia ajustada para la edad. Para evitar la sobreestimación de episodios infecciosos, un segundo evento infeccioso en el mismo sujeto se definió como sepsis después de un mínimo de seis días de estabilidad hemodinámica sin antibióticos. Se definió a la población con inmunodeficiencia bajo los siguientes diagnósticos: trasplante de órgano sólido, trasplante de células madre hematopoyéticas, inmunodeficiencia primaria, tumores sólidos, síndrome nefrótico, síndrome de Down, desnutrición severa, inmunodeficiencia hematológica (i.e. leucemia, linfohistiocitosis hemofagocítica) e inmunodeficiencia inducida por drogas.

Las variables cuantitativas se describieron bajo análisis de frecuencias y porcentajes. Para realizar una comparación estadística entre grupos se utilizó la prueba de Kruskal-Wallis. Se utilizaron curvas ROC para evaluar los valores de corte de PCT. Se asumió significancia estadística si la hipótesis nula podía rechazarse con un valor de $p<0.05$. El análisis 
Rev Latin Infect Pediatr. 2021; 34 (1): 27-33

estadístico fue realizado con el programa SPSS v. 21 (IBM Corp., EUA).

\section{RESULTADOS}

Se realizaron 41,836 hemocultivos de enero de 2011 hasta abril de 2018, 5,059 cultivos (12.09\%) fueron positivos. Los niveles de PCT se documentaron en 311 sujetos; se excluyeron 191 casos por aislamiento polimicrobiano $(n=2)$, contaminación $(n=68)$ y duplicación $(n=121)$ de hemocultivos. La muestra total incluyó 120 niños (Figura 1). La edad media fue de 55 meses, $54 \%$ fueron masculinos y $44.2 \%$ presentaban una inmunodeficiencia.

La media de PCT fue $15.3 \mu \mathrm{g} / \mathrm{L}$. En niños con inmunodeficiencia la media fue de $26.68 \mu \mathrm{g} / \mathrm{L}$, en comparación con $8.78 \mu \mathrm{g} / \mathrm{L}$ en inmunocompetentes $(p<0.05)$. La distribución de los niveles de PCT se muestra en la Tabla 1. Las inmunodeficiencias más frecuentes fueron hematológicas $(53.8 \%$ ) y primarias $(9.6 \%)$. Los sujetos con inmunodeficiencia hematológica tuvieron la media más alta de PCT (31.4 $\mu \mathrm{g} / \mathrm{L})$, seguido por niños con trasplante de células hematopoyéticas $(16.9 \mu \mathrm{g} / \mathrm{L})$; sin embargo, esto no fue estadísticamente significativo $(p \geq 0.05)$.
Los niveles de PCT por microorganismo se muestran en la Tabla 2. El grupo de microorganismos aislados más frecuentes fueron BGN $(65.8 \%)$, seguido de CGP (24.2\%) y levaduras (10\%). Los sujetos con sepsis por BGN tuvieron la media más alta en el valor de PCT $(18.2 \pm 34.2 \mu \mathrm{g} / \mathrm{L})$, en comparación con CGP $(13.1 \pm 36 \mu \mathrm{g} / \mathrm{L})$ y levaduras $(1.9 \pm 1.69$ $\mu \mathrm{g} / \mathrm{L})$. Encontramos una diferencia estadísticamente significativa en la media de valores de PCT entre los grupos de microorganismos $(p<0.01)$. La bacteria con la media más alta de PCT fue el estreptococo $\beta$-hemolítico $(39.3 \mu \mathrm{g} / \mathrm{L})$, seguido por Klebsiella pneumoniae $(28.4 \mu \mathrm{g} / \mathrm{L})$ y Streptococcus pneumoniae $(25.8 \mu \mathrm{g} / \mathrm{L})$.

Las curvas ROC se utilizaron para evaluar la efectividad diagnóstica de la PCT en la predicción de la positividad de un hemocultivo (Figura 2). Utilizando un valor de corte de $0.5 \mu \mathrm{g} / \mathrm{L}$, encontramos una sensibilidad de $58 \%$ y especificidad de $35 \%$, con un área bajo la curva (AUC) de 0.63 (95\% IC, 0.51-0.76). Se observaron valores superiores en inmunocomprometidos, con sensibilidad de $82 \%$ y especificidad de $53 \%$ (AUC 0.63, 95\% IC, 0.45-0.81). La sensibilidad y especificidad por microorganismo fueron 78 y $53 \%$ para CGP, respectivamente (AUC

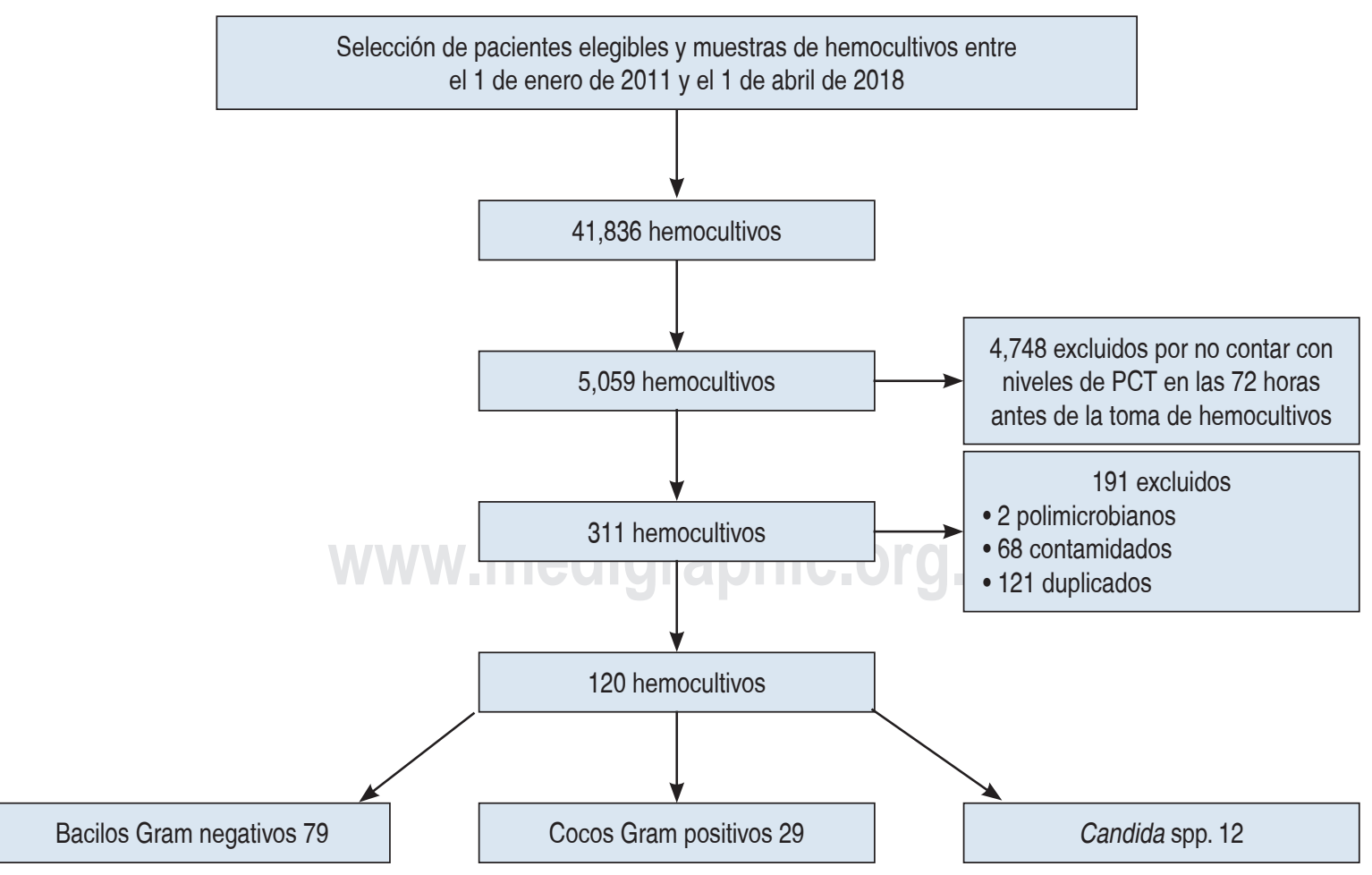

Figura 1: Criterios de inclusión y exclusión. 
A

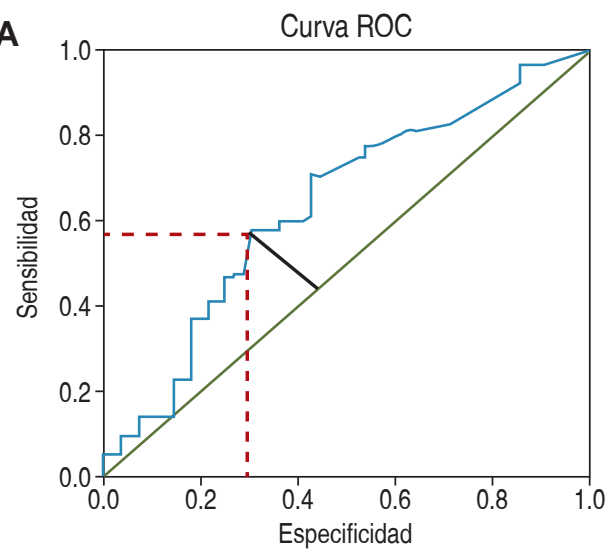

C

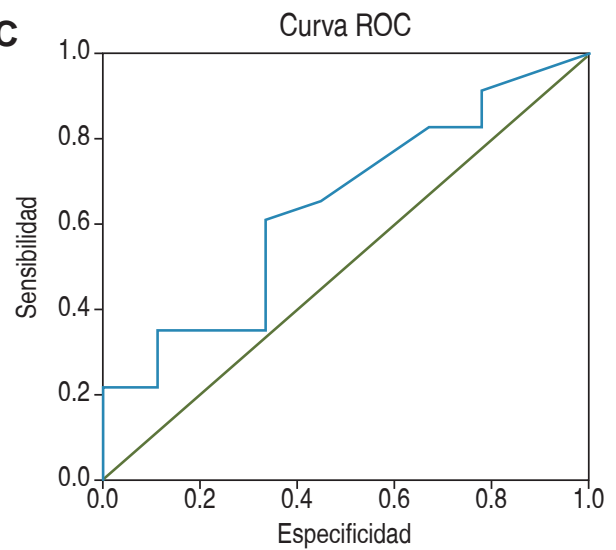

B

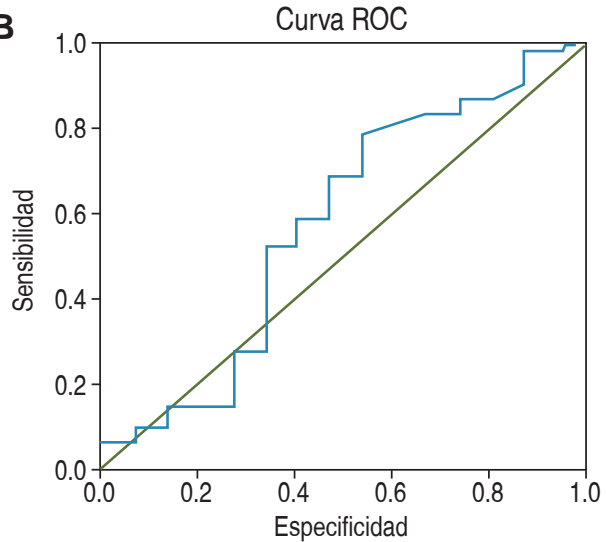

D

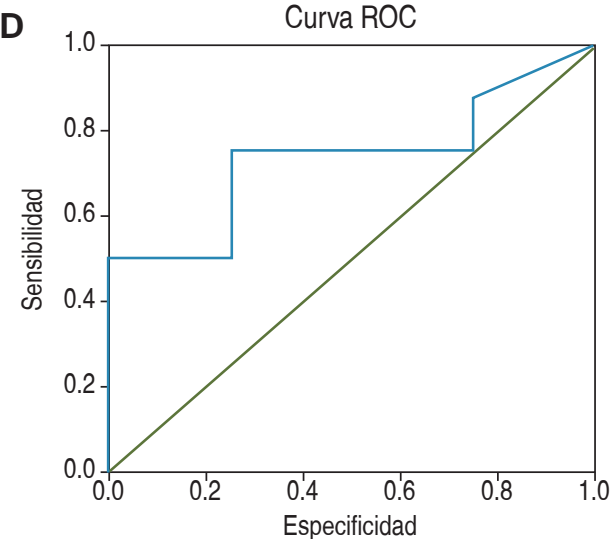

Figura 2:

A) Curva ROC de PCT, AUC: $0.63(95 \%$ IC, $0.51-0.76)$.

B) Curva ROC de PCT para CGP, AUC: 0.58 (95\% IC, $0.40-0.76)$. C) Curva ROC para BGN, AUC: $0.64(95 \%$ IC, $0.42-0.85)$. D) Curva ROC para levaduras, AUC: $0.73(95 \%$ IC, $0.44-0.10)$.
$0.581,95 \%$ IC, 0.40-0.76), comparado con 60.9 y $33.3 \%$ para BGN (AUC 0.640, 95\% IC, 0.42-0.85). Para levaduras, encontramos una sensibilidad de $75 \%$ y especificidad de $25 \%$ (AUC $0.734,95 \%$ IC, 0.44-1.00).

El análisis de subgrupos demostró una sensibilidad de $87.5 \%$ y especificidad de $16.7 \%$ para predecir positividad de un hemocultivo mediante PCT en infección por BGN de pacientes inmunocomprometidos (AUC 0.90, 95\% IC, 0.74-1.00). En el caso de infección por CGP, se encontró una sensibilidad de $78.8 \%$ y especificidad de $22.2 \%$ (AUC $0.74,95 \%$ IC, 0.54-0.94).

\section{DISCUSIÓN}

Aunque se ha descrito previamente a la PCT como un biomarcador para sepsis, el conocimiento actual de los niveles de PCT para predecir la positividad de hemocultivos es escaso, especialmente en niños. ${ }^{12,13}$ En nuestro estudio se incluyeron 120 pacientes durante un periodo de siete años, que describe a la
PCT como una herramienta efectiva para predecir la positividad de hemocultivos en niños con sepsis, especialmente en inmunocomprometidos con infecciones por BGN.

Según los datos obtenidos mediante este estudio, la media del valor de PCT en las infecciones por BGN fue significativamente más alta en comparación con el valor de las infecciones por CGP y levaduras. Encontramos una sensibilidad de $75 \%$ y especificidad de $53 \%$ al utilizar la PCT como predictor en infecciones por BGN en niños. Estudios previos en adultos demostraron resultados similares. ${ }^{14-16}$ La mediana del valor de PCT en la población adulta con infección por BGN se ha descrito en $26.1,25.1$ y $7.47 \mathrm{ng} / \mathrm{mL}$, comparable con nuestros resultados con un valor de $18.2 \mu \mathrm{g} / \mathrm{L} .{ }^{17-19}$ Un reporte previo en población adulta realizado por Shuhua y colaboradores ${ }^{19}$ demostró una sensibilidad y especificidad de 63.9 y $93.3 \%$ para las infecciones por BGN, al usar un valor de corte de $3.11 \mathrm{ng} / \mathrm{mL}$. Yan y su grupo ${ }^{1}$ y Watanabe con su equipo de investigadores ${ }^{20}$ describieron una sensibilidad similar en infecciones por BGN, con una 
Rev Latin Infect Pediatr. 2021; 34 (1): 27-33

sensibilidad de 72.5 y $74.5 \%$, respectivamente. Un análisis de subgrupos de nuestro estudio demuestra una sensibilidad de $87.5 \%$ utilizando la PCT para predecir positividad de hemocultivos en infección por BGN en niños inmunocomprometidos (AUC 0.90, $95 \%$ IC, 0.748-1.00), hallazgo que no se ha descrito previamente en niños.

El rol de la PCT como predictor de CGP en hemocultivos, principalmente en infecciones causadas por Staphylococcus sp., fue evaluado por Shomali y colaboradores, ${ }^{21}$ el cual describe valores más altos en la media de PCT en infecciones por $S$. aureus en comparación con estafilococos coagulasa-negativos $(0.85 \mu \mathrm{g} / \mathrm{L}$ vs $0.26 \mu \mathrm{g} / \mathrm{L})$. En nuestro estudio, al comparar los niveles de PCT entre $S$. aureus y estafilococos coagulasa-negativos, encontramos niveles más elevados de PCT en infecciones por estafilococos coagulasa-negativos (17.3 vs $0.8 \mathrm{ng} / \mathrm{mL}$ ), un resultado diferente al reportado por Shomali. Esta diferencia puede ser atribuida a la frecuencia mayor de aislamientos de estafilococos coagulasa-negativos en nuestro hospital; sin embargo, se requieren estudios con un tamaño de muestra mayor para analizar estos datos.

Los estudios que analizan los niveles de PCT como biomarcador para infecciones fúngicas invasivas por Candida spp. son escasos y muestran datos contradictorios. ${ }^{22-25}$ Shuhua y colaboradores, ${ }^{20}$ Miglietta y su grupo, ${ }^{26}$ Oussalah con su equipo ${ }^{27}$ y Leli y colaboradores ${ }^{28}$ reportan una mediana de PCT en infecciones por Candida spp. de 0.6, 0.5 y $1 \mathrm{ng} / \mathrm{mL}$

\begin{tabular}{lcc}
\multicolumn{3}{c}{$\begin{array}{c}\text { Tabla 1: Niveles de procalcitonina por } \\
\text { tipo de inmunodeficiencia. }\end{array}$} \\
& $n(\%)$ & $\begin{array}{c}\text { Media de nivel de } \\
\text { PCT }(\mu \mathrm{g} / \mathrm{L})\end{array}$ \\
Tipo de ID* & 2.4 \\
\hline TOS & $2(3.8)$ & 3.8 \\
IDP & $5(9.6)$ & 31.4 \\
Hematología & $28(53.8)$ & 23.8 \\
TCMH & $2(3.8)$ & 9.3 \\
Tumor sólido & $5(9.6)$ & 16.9 \\
Síndrome nefrótico & $3(5.8)$ & 1.14 \\
Síndrome de Down & $3(5.8)$ & 1.9 \\
Desnutrición severa & $3(5.8)$ & 180 \\
Drogas & $1(1.9)$ & \\
& & \\
p = 0.15. & & \\
* Análisis Kruskal-Wallis & \\
ID = inmunodeficiencia; IDP = inmunodeficiencia primaria; TOS = trasplante \\
de órgano sólido; TCMH = trasplante de células madre hematopoyéticas.
\end{tabular}

\begin{tabular}{|c|c|c|c|}
\hline \multicolumn{4}{|c|}{$\begin{array}{l}\text { Tabla 2. Niveles procalcitonina de } \\
\text { acuerdo con el patógeno aislado }\end{array}$} \\
\hline Patógeno & $n(\%)$ & $\begin{array}{l}\text { Media de } \\
\text { PCT ( } \mu \mathrm{g} / \mathrm{L})\end{array}$ & Rango \\
\hline $\begin{array}{l}\text { Bacterias Gram } \\
\text { negativas }\end{array}$ & 79 & 18.2 & $0.3-243.4$ \\
\hline Acinetobacter spp & $3(2.5)$ & 3.4 & $0.99-8.16$ \\
\hline Burkholderia cepacia & $2(1.7)$ & 0.1 & $0.09-0.21$ \\
\hline Enterobacter cloacae & $7(5.8)$ & 14.2 & $0.18-45.8$ \\
\hline E. coli & $18(15)$ & 16.9 & $0.26-93.6$ \\
\hline Klebsiella spp & $25(20.8)$ & 28.4 & $0.46-243.4$ \\
\hline Salmonella spp & $3(2.5)$ & 13.7 & $0.43-34.9$ \\
\hline $\begin{array}{l}\text { Stenotrophomonas } \\
\text { maltophiliia }\end{array}$ & $6(5)$ & 7.9 & $0.03-40$ \\
\hline $\begin{array}{l}\text { Pseudomonas } \\
\text { aeruginosa }\end{array}$ & $15(12.5)$ & 15.1 & $0.33-76.6$ \\
\hline $\begin{array}{l}\text { Bacterias Gram } \\
\text { positivas }\end{array}$ & 29 & 13.1 & $0.03-180$ \\
\hline Enterococcus spp & $3(2.5)$ & 1 & $0.4-2.2$ \\
\hline $\begin{array}{l}\text { Staphylococcus } \\
\text { aureus }\end{array}$ & $8(6.7)$ & 0.8 & $0.7-3$ \\
\hline $\begin{array}{l}\text { Estafilococos } \\
\text { coagulasa-negativos }\end{array}$ & $11(9.2)$ & 17.3 & $0.1-180$ \\
\hline $\begin{array}{l}\text { Staphylococcus } \\
\text { lugdunensis }\end{array}$ & $2(1.7)$ & 5.1 & $0.03-101$ \\
\hline $\begin{array}{l}\text { Estreptococo } \\
\beta \text {-hemolítico }\end{array}$ & $3(2.5)$ & 39.3 & $7.3-65$ \\
\hline $\begin{array}{l}\text { Streptococcus } \\
\text { pneumoniae }\end{array}$ & $2(1.7)$ & 25.8 & $1.2-50.4$ \\
\hline Hongos & 12 & 1.9 & $0.2-5.5$ \\
\hline Candida spp & $12(10)$ & 1.9 & $0.2-5.5$ \\
\hline
\end{tabular}

respectivamente. En otro estudio por Thomas-Ruddel y colaboradores ${ }^{18}$ se describe una mediana más elevada, en $4.7 \mathrm{ng} / \mathrm{mL}$. De manera consistente con estudios previos en poblaciones adultas, reportamos niveles menores de PCT en infecciones fúngicas por Candida sp. en comparación con infecciones bacterianas. En un estudio previo por Cortegiani y su grupo, ${ }^{25}$ usando los niveles de PCT para predecir infecciones por Candida sp., describieron una sensibilidad de $86.8 \%$. Estos resultados fueron similares a los nuestros (sensibilidad de 75\%). Aunque la identificación de especies de Candida no fue realizada en nuestro estudio, otros autores no han encontrado diferencia entre los niveles de PCT de acuerdo con las diferentes especies.

El conocimiento actual del rol de la PCT en niños inmunocomprometidos es escaso. ${ }^{29-34}$ Existen estudios 
previos en niños con cáncer que mostraron que la PCT es un marcador efectivo para sepsis durante un episodio de fiebre y neutropenia; sin embargo, ninguno de ellos evaluó el rol de la PCT para predecir la positividad de un hemocultivo..$^{30,34}$ Nuestro estudio reporta una sensibilidad de $87.5 \%$ para predecir la positividad de un hemocultivo en infecciones por BGN, lo que pudiera apoyar el uso de PCT como una herramienta útil en la práctica clínica. Además, los niveles de PCT se vieron incrementados en los diferentes casos de inmunodeficiencia. De acuerdo con la media de PCT, encontramos una diferencia estadísticamente significativa entre inmunocomprometidos $(26.68 \mu \mathrm{g} / \mathrm{L})$ e inmunocompetentes $(8.78$ $\mu \mathrm{g} / \mathrm{L})(\mathrm{p}<0.05)$. Estos resultados contrastan con lo reportado por Al-Nawas y Shah en adultos. ${ }^{33}$

Nuestro estudio tiene limitaciones, un diseño prospectivo ayudaría a tener mejor control de las variables e incluir una muestra mayor para evadir la heterogeneidad de los casos. Asimismo, las mediciones de PCT no fueron seriadas, lo cual nos podría permitir analizar el comportamiento de la PCT.

Nuestro estudio encontró que la PCT puede ser una herramienta útil para predecir la positividad de hemocultivos en niños con sepsis, especialmente en inmunocomprometidos con infecciones por BGN. Se necesitan más estudios prospectivos para expandir la evidencia disponible sobre el uso de la PCT en niños como un predictor de especies en hemocultivos.

\section{REFERENCIAS}

1. Yan ST, Sun LC, Jia HB, Gao W, Yang JP, Zhang GQ. Procalcitonin levels in bloodstream infections caused by different sources and species of bacteria. Am J Emerg Med. 2017; 35 (4): 579-583.

2. Liu HH, Zhang MW, Guo JB, Li J, Su L. Procalcitonin and $\mathrm{C}$-reactive protein in early diagnosis of sepsis caused by either Gram negative or Gram positive bacteria. Ir J Med Sci. 2017; 186 (1): 207-212.

3. Balamuth F, Weiss SL, Neuman MI, Scott H, Brady PW, Paul R et al. Pediatric severe sepsis in U.S. children's hospitals. Pediatr Crit Care Med. 2014; 15 (9): 798-805.

4. Singer M, Deutschman CS, Seymour CW, Shankar-Hari M, Annane D, Bauer $\mathrm{M}$ et al. The third international consensus definitions for sepsis and septic shock (Sepsis-3). JAMA. 2016; 315 (8): 801-810.

5. Hawiger J, Veach RA, Zienkiewicz J. New paradigms in sepsis: From prevention to protection of failing microcirculation. $J$ Thromb Haemost. 2015; 13 (10): 1743-1756.

6. Simon L, Gauvin F, Amre DK, Saint-Louis P, Lacroix J. Serum procalcitonin and C-reactive protein levels as markers of bacterial infection: a systematic review and meta-analysis. Clin Infect Dis. 2004; 39 (2): 206-217.
7. Casado Flores J, Blanco Quirós A. Procalcitonina: un nuevo marcador de infección bacteriana. An Pediatría. 2001; 54 (1): 69-73.

8. Cha JK, Kwon KH, Byun SJ, Ryoo SR, Lee JH, Chung JW et al. Clinical value of procalcitonin for suspected nosocomial bloodstream infection. Korean J Intern Med. 2018; 33 (1): 176-184.

9. Riedel S, Melendez JH, An AT, Rosenbaum JE, Zenilman JM. Procalcitonin as a marker for the detection of bacteremia and sepsis in the emergency department. Am J Clin Pathol. 2011; 135 (2): 182-189.

10. Sager R, Kutz A, Mueller B, Schuetz P. Procalcitonin-guided diagnosis and antibiotic stewardship revisited. BMC Med. 2017; 15 (1): 15.

11. Díez-Padrisa N, Bassat Q, Morais L, O'Callaghan-Gordo C, Machevo S, Nhampossa $T$ et al. Procalcitonin and C-reactive protein as predictors of blood culture positivity among hospitalized children with severe pneumonia in Mozambique. Trop Med Int Heal. 2012; 17 (9): 11001107.

12. Lanziotti VS, Póvoa P, Soares M, Silva JR, Barbosa AP, Salluh JI. Use of biomarkers in pediatric sepsis: literature review. Rev Bras Ter Intensiva. 2016; 28 (4): 472-482.

13. Pontrelli G, De Crescenzo F, Buzzetti R, Jenkner A, Balduzzi $\mathrm{S}$, Calo Carducci $\mathrm{F}$ et al. Accuracy of serum procalcitonin for the diagnosis of sepsis in neonates and children with systemic inflammatory syndrome: a meta-analysis. BMC Infect Dis. 2017; 17 (1): 302.

14. Kim MH, Lim G, Kang SY, Lee WI, Suh JT, Lee HJ. Utility of procalcitonin as an early diagnostic marker of bacteremia in patients with acute fever. Yonsei Med J. 2011; 52 (2): 276-281.

15. Carrol ED, Mankhambo LA, Jeffers G, Parker D, Guiver M, Newland $P$ et al. The diagnostic and prognostic accuracy of five markers of serious bacterial infection in Malawian children with signs of severe infection. PLoS One. 2009; 4 (8): e6621.

16. Charles PE, Ladoire S, Aho S, Quenot JP, Doise JM, Prin S et al. Serum procalcitonin elevation in critically ill patients at the onset of bacteremia caused by either Gram negative or Gram positive bacteria. BMC Infect Dis. 2008; 8: 38.

17. Thomas-Ruddel DO, Poidinger B, Kott M, Weiss M, Reinhart $\mathrm{K}$, Bloos F; MEDUSA study group. Influence of pathogen and focus of infection on procalcitonin values in sepsis patients with bacteremia or candidemia. Crit Care. 2018; 22 (1): 128

18. Bassetti M, Russo A, Righi E, Dolso E, Merelli M, Cannarsa $\mathrm{N}$ et al. Comparison between procalcitonin and C-reactive protein to predict blood culture results in ICU patients. Crit Care. 2018; 22 (1): 252

19. Li S, Rong H, Guo Q, Chen Y, Zhang G, Yang J. Serum procalcitonin levels distinguish Gram-negative bacterial sepsis from Gram-positive bacterial and fungal sepsis. J Res Med Sci. 2016; 21: 39.

20. Watanabe Y, Oikawa N, Hariu M, Fuke R, Seki M. Ability of procalcitonin to diagnose bacterial infection and bacteria types compared with blood culture findings. Int J Gen Med. 2016; 9: 325-331.

21. Shomali W, Hachem R, Chaftari AM, Bahu R, Helou GE, Jiang $Y$ et al. Can procalcitonin differentiate Staphylococcus aureus from coagulase-negative staphylococci in clustered gram-positive bacteremia? Diagn Microbiol Infect Dis. 2013; 76 (2): 158-161. 
Rev Latin Infect Pediatr. 2021; 34 (1): 27-33

22. Martini A, Gottin L, Menestrina N, Schweiger V, Simion D, Vincent JL. Procalcitonin levels in surgical patients at risk of candidemia. J Infect. 2010; 60 (6): 425-430.

23. Brodská H, Malícková K, Adámková V, Benáková H, St’astná MM, Zima T. Significantly higher procalcitonin levels could differentiate Gram-negative sepsis from Gram-positive and fungal sepsis. Clin Exp Med. 2013; 13 (3): 165-170.

24. Montagna MT, Coretti C, Caggiano G. Procalcitonin: a possible marker of invasive fungal infection in high risk patients? J Prev Med Hyg. 2011; 52 (1): 38-39.

25. Cortegiani A, Russotto V, Montalto F, Foresta G, Accurso G, Palmeri $C$ et al. Procalcitonin as a marker of Candida species detection by blood culture and polymerase chain reaction in septic patients. BMC Anesthesiol. 2014; 14: 9.

26. Miglietta F, Faneschi ML, Lobreglio G, Palumbo C, Rizzo $\mathrm{A}$, Cucurachi $\mathrm{M}$ et al. Procalcitonin, $\mathrm{C}$-reactive protein and serum lactate dehydrogenase in the diagnosis of bacterial sepsis, SIRS and systemic candidiasis. Infez Med. 2015; 23 (3): 230-237.

27. Oussalah A, Ferrand J, Filhine-Tresarrieu P, Aissa N, Aimone-Gastin I, Namour F et al. Diagnostic accuracy of procalcitonin for predicting blood culture results in patients with suspected bloodstream infection: an observational study of 35,343 consecutive patients (A STROBECompliant Article). Medicine (Baltimore). 2015; 94 (44): e1774.

28. Leli C, Ferranti M, Moretti A, AI Dhahab ZS, Cenci E, Mencacci A. Procalcitonin levels in gram-positive, gramnegative, and fungal bloodstream infections. Dis Markers. 2015; 2015: 701480.

29. Nielsen MJ, Baines $P$, Jennings R, Siner S, KolamunnageDona R, Newland $P$ et al. Procalcitonin, C-reactive protein, neutrophil gelatinase-associated lipocalin, resistin and the APTT waveform for the early diagnosis of serious bacterial infection and prediction of outcome in critically ill children. PLoS One. 2021; 16 (2): e0246027.

30. Fleischhack G, Cipic D, Juettner J, Hasan C, Bode U. Procalcitonin-a sensitive inflammation marker of febrile episodes in neutropenic children with cancer. Intensive Care Med. 2000; 26 Suppl 2: S202-S211.

31. Fleischhack G, Kambeck I, Cipic D, Hasan C, Bode U. Procalcitonin in paediatric cancer patients: Its diagnostic relevance is superior to that of C-reactive protein, interleukin 6 , interleukin 8 , soluble interleukin 2 receptor and soluble tumour necrosis factor receptor II. Br J Haematol. 2000; 111 (4): 1093-1102.

32. Staehler M, Hammer C, Meiser B, Reichart B. Procalcitonin a new marker for differential diagnosis of acute rejection and bacterial infection in heart transplantation. Transplant Proc. 1997; 29 (1-2): 584-585.

33. al-Nawas B, Shah PM. Procalcitonin in patients with and without immunosuppression and sepsis. Infection. 1996; 24 (6): 434-436.

34. Arif T, Phillips RS. Updated systematic review and metaanalysis of the predictive value of serum biomarkers in the assessment and management of fever during neutropenia in children with cancer. Pediatr Blood Cancer. 2019; 66 (10): e27887.

Correspondencia:

Abiel Homero Mascareñas-De los Santos

Departamento de Pediatría, División de Infectología,

Hospital Universitario «Dr. José Eleuterio González».

Avenida Francisco I. Madero,

Col. Mitras Centro, 64460,

Monterrey, México.

Tel y Fax: 81 8389-1111

E-mail: a_mascarenas@hotmail.com 